\title{
Multigrid and Domain Decomposition Methods for Electrostatics Problems
}

\author{
Michael Holst and Faisal Saied
}

\begin{abstract}
We consider multigrid and domain decomposition methods for the numerical solution of electrostatics problems arising in biophysics. We compare multigrid methods designed for discontinuous coefficients with domain decomposition methods, including comparisons of standard multigrid methods, algebraic multigrid methods, additive and multiplicative Schwarz domain decomposition methods, and acceleration of multigrid and domain decomposition methods with conjugate gradient methods. As a test problem, we consider a linearization of the Poisson-Boltzmann equation, which describes the electrostatic potential of a large complex biomolecule lying in an ionic solvent.
\end{abstract}

\section{Introduction}

In recent years, multigrid (MG) and domain decomposition (DD) methods have been used extensively as tools for obtaining approximations to solutions of partial differential equations (see, for example, the references in [15]). In this paper, we consider MG and DD methods for the numerical solution of the Poisson-Boltzmann equation, which describes the electrostatic potential of a large complex biomolecule lying in an ionic solvent (see, for example, $[\mathbf{3}, \mathbf{1 4}]$ for an overview). We compare MG methods designed for discontinuous coefficients with DD methods, when applied to a two-dimensional, linearized Poisson-Boltzmann equation. Several approaches are considered, including standard MG methods, algebraic MG methods, additive and multiplicative Schwarz methods, and the acceleration of MG and DD methods with conjugate gradient (CG) methods.

\section{Background Material}

The nonlinear Poisson-Boltzmann equation (PBE) for the dimensionless electrostatic potential $u(\mathbf{r})=e_{c} \phi(\mathbf{r}) k_{B}^{-1} T^{-1}$ has the form:

$-\nabla \cdot(\epsilon(\mathbf{r}) \nabla u(\mathbf{r}))+\bar{\kappa}^{2} \sinh (u(\mathbf{r}))=\left(\frac{4 \pi e_{c}^{2}}{k_{B} T}\right) \sum_{i=1}^{N_{m}} z_{i} \delta\left(\mathbf{r}-\mathbf{r}_{i}\right), \quad \mathbf{r} \in \mathbb{R}^{3}, \quad \Phi(\infty)=0$,

1991 Mathematics Subject Classification. Primary 65N30; Secondary 65F10.

The first author was supported in part by DOE Grant No. DOE DE-FG02-91ER25099.

The second author was supported in part by NSF Grant No. NSF ASC 9209502 RIA..

This paper is in final form and no version of it will be submitted for publication elsewhere. 
where $\phi(\mathbf{r})$ denotes the electrostatic potential at field position $\mathbf{r}$. The coefficients appearing in the equation are necessarily discontinuous by several orders of magnitude, describing both the molecular surface $(\epsilon(\mathbf{r}))$ and an ion-exclusion layer $(\bar{\kappa}(\mathbf{r}))$ around the molecule. The placement and magnitude of atomic charges are represented by the source terms involving the delta-functions.

Using known analytical solutions for special situations, approximate boundary conditions are obtained for a finite domain $\Omega \subset \mathbb{R}^{3}$ containing the molecule and some of the surrounding solvent; the problem is then solved as a finite-boundary problem. A linearized form of the equation is often solved as an approximation to the full nonlinear problem $[\mathbf{5}, \mathbf{9}, \mathbf{1 2}]$. Damped-inexact-Newton methods combined with algebraic MG methods have been shown to be efficient and robust for the full nonlinear problem $[\mathbf{7}, \mathbf{8}]$.

\section{Multigrid Methods}

Consider a nested sequence of finite-dimensional Hilbert spaces

$$
\mathcal{H}_{1} \subset \mathcal{H}_{2} \subset \cdots \subset \mathcal{H}_{J}=\mathcal{H}
$$

each with an associated inner-product $(\cdot, \cdot)_{k}$ inducing the norm $\|\cdot\|_{k}=(\cdot, \cdot)_{k}^{1 / 2}$. Also associated with each $\mathcal{H}_{k}$ is an operator $A_{k}$, assumed to be SPD with respect to $(\cdot, \cdot)_{k}$. The spaces $\mathcal{H}_{k}$, which may be finite element function spaces or simply $\mathbf{R}^{n_{k}}$ (where $\left.n_{k}=\operatorname{dim}\left(\mathcal{H}_{k}\right)\right)$, are connected by prolongation operators $I_{k-1}^{k} \in \mathbf{L}\left(\mathcal{H}_{k-1}, \mathcal{H}_{k}\right)$, and restriction operators $I_{k}^{k-1} \in \mathbf{L}\left(\mathcal{H}_{k}, \mathcal{H}_{k-1}\right)$. It is assumed that the operators satisfy variational conditions:

$$
A_{k-1}=I_{k}^{k-1} A_{k} I_{k-1}^{k}, \quad I_{k}^{k-1}=\left(I_{k-1}^{k}\right)^{T} .
$$

These conditions hold naturally in the finite element setting, and are imposed directly in algebraic MG methods.

Given $B \approx A^{-1}$ in the space $\mathcal{H}$, the basic linear method constructed from the preconditioned system $B A u=B f$ has the form:

$$
u^{n+1}=u^{n}-B A u^{n}+B f=(I-B A) u^{n}+B f .
$$

Now, given some $B$, or some procedure for applying $B$, we can either formulate a linear method using $E=I-B A$, or employ a CG method for $B A u=B f$ if $B$ is SPD.

The recursive formulation of MG methods has been well-known for more than fifteen years; mathematically equivalent forms of the method involving product error propagators have been recognized and exploited theoretically only very recently. In particular, it can be shown $[\mathbf{2}, \mathbf{1 1}]$ that if the conditions $(3.1)$ hold, then the MG error propagator can be factored as:

$$
E_{J}=I-B_{J} A_{J}=\left(I-T_{J ; J}\right)\left(I-T_{J ; J-1}\right) \cdots\left(I-T_{J ; 1}\right),
$$

where:

$$
\begin{gathered}
I_{k-i}^{k}=I_{k-1}^{k} I_{k-2}^{k-1} \cdots I_{k-i+1}^{k-i+2} I_{k-i}^{k-i+1}, \quad I_{k}^{k-i}=I_{k-i+1}^{k-i} I_{k-i+2}^{k-i+1} \cdots I_{k-1}^{k-2} I_{k}^{k-1}, \quad I_{k}^{k}=I, \\
T_{J ; 1}=I_{1}^{J} A_{1}^{-1} I_{J}^{1} A_{J}, \quad T_{J ; k}=I_{k}^{J} R_{k} I_{J}^{k} A_{J}, \quad k=2, \ldots, J,
\end{gathered}
$$

where $R_{k} \approx A_{k}^{-1}$ is the "smoothing" operator employed in each space $\mathcal{H}_{k}$. We make this remark simply to stress the similarities between MG methods and certain DD methods discussed in the next section. 
For problems such as the Poisson-Boltzmann equation, the coefficient discontinuities are complex, and they may not lie on coarse mesh element boundaries as required for accurate finite element approximation (and as required for validity of finite element error estimates). MG methods typically perform badly, and even the regularity-free MG convergence theory [2] is invalid.

Possible approaches include coefficient averaging methods (cf. [1]) and the explicit enforcement of the conditions (3.1) (cf. $[\mathbf{1}, \mathbf{6}, \mathbf{1 3}])$. By introducing a symbolic stencil calculus and employing Maple or Mathematica, the conditions (3.1) can be enforced algebraically in an efficient way for certain types of sparse matrices; details may be found for example in [7].

\section{Domain Decomposition Methods}

DD methods were first proposed by H.A. Schwarz as a theoretical tool for studying elliptic problems on complicated domains, constructed as the union of simple domains. An interesting early reference not often mentioned is [10], containing both analysis and numerical examples, and references to the original work by Schwarz.

Given a domain $\Omega$ and coarse triangulation by $N$ regions $\left\{\Omega_{k}\right\}$ of mesh size $H$, we refine (several times) to obtain a fine mesh of size $h$. The regions defined by the initial triangulation $\Omega_{k}$ are then extended by $\delta_{k}$ to form the "overlapping subdomains" $\Omega_{k}^{\prime}$. Now, let $V$ and $V_{0}$ denote the finite element spaces associated with the $h$ and $H$ triangulation of $\Omega$, respectively. The variational problem in $V$ has the form:

Find $u \in V$ such that $a(u, v)=f(v), \quad \forall v \in V$.

The form $a(\cdot, \cdot)$ is bilinear, symmetric, coercive, and bounded, whereas $f(\cdot)$ is linear and bounded. Therefore, through the Riesz representation theorem we can associate with the above problem an abstract operator equation $A u=f$, where $A$ is SPD.

DD methods can be seen as iterative methods for solving the above operator equation, involving approximate projections of the error onto subspaces of $V$ associated with the overlapping subdomains $\Omega_{k}^{\prime}$. To be more specific, let $V_{k}=H_{0}^{1}\left(\Omega_{k}^{\prime}\right) \cap V$, $k=1, \ldots, N$; it is not difficult to show that $V=V_{1}+\cdots+V_{N}$, where a coarse space $V_{0}$ may also be included in the sum.

As with MG methods, we denote $A_{k}$ as the restriction of the operator $A$ to the space $V_{k}$. Algebraically, it can be shown that $A_{k}=N_{k}^{T} A N_{k}$, where $N_{k}$ is the natural inclusion in $\mathbb{R}^{n_{k}}$, and $N_{k}^{T}$ is the corresponding projection. In other words, DD methods automatically satisfy the variation conditions (3.1) in the subspaces $V_{k}, k \neq 0$. Now, if $R_{k} \approx A_{k}^{-1}$, we can define the approximate $A$-orthogonal projector from $V$ onto $V_{k}$ as $T_{k}=N_{k} R_{k} N_{k}^{T} A$. An overlapping DD method can be written as a basic linear method as in equation (3.2), where the multiplicative Schwarz error propagator $E$ is:

$$
E=I-B A=\left(I-T_{N}\right)\left(I-T_{N-1}\right) \cdots\left(I-T_{0}\right) .
$$

The additive Schwarz error propagator $E$ is:

$$
E=I-B A=I-\omega\left(T_{0}+T_{1}+\cdots+T_{N}\right) .
$$

An additive-multiplicative variant has been proposed in [4], which takes only the coarse space projection an additive term in the following way:

$$
E=I-B A=\left(I-T_{N}\right)\left(I-T_{N-1}\right) \cdots\left(I-T_{1}\right)-\omega T_{0} .
$$


TABLE 1. Various multigrid and domain decomposition methods.

\begin{tabular}{|l|l|}
\hline \multicolumn{1}{|c|}{ Method } & \multicolumn{1}{|c|}{ Description } \\
\hline MG & FEM-based MG, weighted Jacobi smoothing \\
M & multiplicative Schwarz \\
A & additive Schwarz (with a damping parameter) \\
AM & multiplicative Schwarz with additive coarse term \\
\hline CGMG & CG preconditioned with MG \\
CGM & CG preconditioned with M \\
CGA & CG preconditioned with A \\
CGAM & CG preconditioned with AM \\
\hline MGG & Algebraic MG, weighted Jacobi smoothing \\
CGMGG & CG preconditioned with algebraic MG \\
\hline
\end{tabular}

This approach decouples the coarse problem in $V_{0}$, allowing it to be solved in parallel with the other subproblems.

\section{An empirical comparison of MG and DD for a 2D PBE}

We now compare several MG and DD methods for a two-dimensional, linearized Poisson-Boltzmann equation. The numerical solution proceeds as follows for two test problems.

In each case, we begin with a simple "triangular" molecule with three point charges. In the first case, we force the molecule surface to align with the coarsest mesh in the MG methods, and to align with the non-overlapped subdomains in the DD methods. In the second case, the discontinuities do not align with the coarsest mesh or the subdomain boundaries (the "non-aligned" case).

Beginning with an initial mesh size $H$, we uniformly refine the mesh five times, yielding a mesh of size $h$. Subdomains are then given a small overlap (one fine mesh triangle, $\delta_{k}=h_{k}$ ). Piecewise linear finite elements are used to discretize the problem in all subdomains for the DD methods, and on all levels for the MG methods; the DD methods employ a coarse space. Figure 1 shows the initial triangulation and a sample overlapped subdomain, and a sample solution. Table 1 gives a key to the remaining figures.

Figure 2 shows the performance of the methods, as a function of CPU time on a SPARC 10, for the aligned problem. The MG methods appear to be the most efficient methods; however, it should be noted that inexact subdomain solvers often lead to improved DD solve times (we employed a sparse direct method). Also, when viewed as error reduction per iteration rather than time in Figure 3, multiplicative Schwarz and multigrid have strikingly similar behavior.

The non-aligned case is illustrated in Figure 4. As expected, the standard MG method fails when the conditions (3.1) are strongly violated. The DD methods remain robust for this problem, whereas the algebraic MG methods appear to be the most efficient. However, note that setup time for the algebraic MG methods (although negligible for this problem) can be quite substantial for some problems. 


\section{Summary and Conclusions}

Convergence theorems for MG and DD methods, applicable in the presence of discontinuous coefficients, rely heavily on the conditions (3.1). Although additional assumptions must be employed to prove that the convergence rate is independent of the meshsize, number of levels, or number of subdomains, very general proofs (although with no rate information) can be given using essentially only (3.1), demonstrating the robustness of this approach.

While the conditions (3.1) are enforced for the algebraic MG methods, they also hold automatically for DD methods, independent of the location of discontinuities in the coefficients. This is not true for the coarse space, which is identical to the MG coarse grid problem; the DD methods appearing in the plots here include a coarse space, but do not explicitly enforce the conditions (3.1) for the coarse problem. If the discontinuities were made worse, the DD methods presented here might also have difficulty with the non-aligned case.

MG and DD methods are comparable sequentially for two-dimensional electrostatics problems. DD methods seem to be naturally more robust, although MG can be made robust and efficient by enforcing the conditions (3.1) explicitly. While the MG methods were generally more efficient, the DD methods offer advantages, such as ease of implementation, as well as parallel implementations. 
FiguRE 1. An overlapping subdomain and a sample solution.

Figure 2. CPU seconds for Case 1. 
FiguRE 3. Iterations for Case 1.

Figure 4. CPU seconds for Case 2. 


\section{References}

[1] R. E. Alcouffe, A. Brandt, J. E. Dendy, Jr., and J. W. Painter, The multi-grid method for the diffusion equation with strongly discontinuous coefficients, SIAM J. Sci. Statist. Comput. 2 (1981), 430-454.

[2] J. H. Bramble, J. E. Pasciak, J. Wang, and J. Xu, Convergence estimates for multigrid algorithms without regularity assumptions, Math. Comp. 57 (1991), 23-45.

[3] J. M. Briggs and J. A. McCammon, Computation unravels mysteries of molecular biophysics, Computers in Physics 6 (1990), 238-243.

[4] X.-C. Cai, An optimal two-level overlapping domain decomposition method for elliptic problems in two and three dimensions, SIAM J. Sci. Statist. Comput. 14 (1993), 239-247.

[5] M. E. Davis and J. A. McCammon, Solving the finite difference linearized Poisson-Boltzmann equation: A comparison of relaxation and conjugate gradient methods, J. Comput. Chem. 10 (1989), 386-391.

[6] J. E. Dendy, Jr., Two multigrid methods for three-dimensional problems with discontinuous and anisotropic coefficients, SIAM J. Sci. Statist. Comput. 8 (1987), 673-685.

[7] M. Holst, Multilevel methods for the Poisson-Boltzmann equation, Ph.D. thesis, Numerical Computing Group, Department of Computer Science, University of Illinois at UrbanaChampaign, 1993, Also published as Tech. Rep. UIUCDCS-R-03-1821.

[8] M. Holst, R. Kozack, F. Saied, and S. Subramaniam, Treatment of electrostatic effects in proteins: Multigrid-based-Newton iterative method for solution of the full nonlinear PoissonBoltzmann equation, Proteins: Structure, Function, and Genetics 18 (1994), 231-245.

[9] M. Holst and F. Saied, Multigrid solution of the Poisson-Boltzmann equation, J. Comput. Chem. 14 (1993), 105-113.

[10] L. V. Kantorovich and V. I. Krylov, Approximate methods of higher analysis, P. Noordhoff, Ltd, Groningen, The Netherlands, 1958.

[11] S. F. McCormick and J. W. Ruge, Unigrid for multigrid simulation, Math. Comp. 41 (1983), $43-62$.

[12] A. Nicholls and B. Honig, A rapid finite difference algorithm, utilizing successive overrelaxation to solve the Poisson-Boltzmann equation, J. Comput. Chem. 12 (1991), 435-445.

[13] J. W. Ruge and K. Stüben, Algebraic multigrid, Multigrid Methods (S. McCormick, ed.), SIAM, 1987, pp. 73-130.

[14] K. A. Sharp and B. Honig, Electrostatic interactions in macromolecules: Theory and applications, Annu. Rev. Biophys. Biophys. Chem. 19 (1990), 301-332.

[15] J. Xu, Iterative methods by space decomposition and subspace correction, SIAM Review 34 (1992), 581-613.

Department of Applied Mathematics and CRPC, California Institute of Technology 217-50, Pasadena, CA 91125

E-mail address: holst@@ama.caltech.edu

Department of Computer Science, University of Illinois at Urbana-Champaign, UrBANA, IL 61801

E-mail address: saied@@cs.uiuc.edu 